\title{
Individualized Anatomic Anterior Cruciate Ligament Reconstruction
}

\author{
Stephen J. Rabuck, M.D., Kellie K. Middleton, M.P.H., Shugo Maeda, M.D., \\ Yoshimasa Fujimaki, M.D., Ph.D., Bart Muller, M.D., Paulo H. Araujo, M.D., and \\ Freddie H. Fu, M.D.
}

\begin{abstract}
Arthroscopic anterior cruciate ligament reconstruction (ACL-R) is a technique that continues to evolve. Good results have been established with respect to reducing anteroposterior laxity. However, these results have come into question because nonanatomic techniques have been ineffective at restoring knee kinematics and raised concerns that abnormal kinematics may impact long-term knee health. Anatomic ACL-R attempts to closely reproduce the patient's individual anatomic characteristics. Measurements of the patient's anatomy help determine graft choice and whether anatomic reconstruction should be performed with a single- or double-bundle technique. The bony landmarks and insertions of the anterior cruciate ligament (ACL) are preserved to assist with anatomic placement of both tibial and femoral tunnels. An anatomic single- or double-bundle reconstruction is performed with a goal of reproducing the characteristics of the native ACL. Long-term outcomes for anatomic ACL reconstruction are unknown. By individualizing ACL-R, we strive to reproduce the patient's native anatomy and restore knee kinematics to improve patient outcomes.
\end{abstract}

A nterior cruciate ligament (ACL) ruptures largely affect the younger, physically active population and, when left untreated, can result in recurrent instability, inability to return to full activity, meniscal tears, and articular cartilage damage. ${ }^{1}$ Given these consequences, surgical reconstruction has been recommended in this population. With the introduction of arthroscopic anterior cruciate ligament reconstruction

From the Department of Orthopaedic Surgery, University of Pittsburgh (S.J.R., K.K.M., S.M., Y.F., B.M., P.H.A., F.H.F.), Pittsburgh, Pennsylvania, U.S.A.; the Department of Orthopaedic Surgery, Graduate School of Medicine, Hirosaki University (S.M.), Hirosaki, Japan; the Department of Orthopaedic Surgery, Showa University School of Medicine (Y.F.), Shinagawa-ku, Japan; and the Department of Orthopaedic Surgery, University of Amsterdam (B.M.), Amsterdam, The Netherlands.

The authors report the following potential conflict of interest or source of funding in relation to this article: Smith \& Nephew.

Received December 13, 2011; accepted December 22, 2011.

Address correspondence to Stephen J. Rabuck, M.D., 3200 S Water St, Pittsburgh, PA 15203, U.S.A. E-mail: rabucksj@upmc .edu

(C) 2012 by the Arthroscopy Association of North America 2212-6287/11837/\$36.00

doi:10.1016/j.eats.2011.12.004
(ACL-R), techniques were developed to safely and reliably place the femoral tunnel in the notch. These techniques led to nonanatomic graft placement. ${ }^{2}$ Conventional, nonanatomic single-bundle (SB) techniques have been largely successful at short-term follow-up. ${ }^{3}$ These results have come into question because recent studies have identified a subset of patients who continue to have subjective knee instability preventing their return to previous activities. ${ }^{4,5}$ Furthermore, long-term evaluation has shown that SB reconstruction does not completely restore knee kinematics and can lead to long-term degenerative changes. ${ }^{6-8}$

Conventional, nonanatomic SB ACL-R may restore anteroposterior stability; however, because the function of the posterolateral (PL) bundle is largely neglected, rotational laxity may persist. ${ }^{9,10}$ These findings appear to be the result of nonanatomic position of the graft. Recently, ACL-R has focused on moving tunnels from the conventional, nonanatomic position to the native insertion of the ACL to restore normal knee kinematics and improve patient outcomes.

The definition of anatomic ACL-R is the functional restoration of the ACL to its native dimensions. ${ }^{11}$ This 
goal may be achieved by either an SB or doublebundle (DB) reconstruction, depending on the patient's individual anatomy. This technical note describes the theory and surgical technique of an anatomic ACL-R.

\section{TECHNIQUE}

\section{Patient Evaluation}

The patient evaluation begins with a comprehensive history and physical examination during which patient expectations, activity level, associated injuries, and comorbidities are assessed. In addition to knee laxity, range of motion is carefully examined and addressed before surgical intervention. Routine imaging includes plain radiographs and magnetic resonance imaging, including oblique sequences oriented in the plane of the ACL (Fig 1). It is imperative to carefully assess the patient's anatomy on preoperative imaging to develop an adequate preoperative plan. Measurements of ACL insertion site size, inclination angle, graft size, and ACL length are critical (Fig 2).

\section{Graft Options}

Anatomic ACL-R is not requisite on one graft type. Depending on the patient's anatomy, various graft types may be beneficial and the surgeon should be adept at graft harvest and preparation. The quadriceps tendon has tremendous versatility for $\mathrm{SB}$ and $\mathrm{DB}$ reconstructions given its superior length and thickness especially when compared with the patellar tendon. ${ }^{12,13}$

\section{Patient Positioning}

The patient is positioned supine on the operating table with a nonsterile tourniquet on the proximal thigh (Video 1). The operative leg is secured within a leg holder and positioned to allow greater than $120^{\circ}$ of flexion (Table 1). The contralateral leg is positioned away from the surgical field in the lithotomy position.

\section{Portals}

Three portals are used for arthroscopy: high anterolateral portal, central portal, and accessory medial portal (Fig 3). ${ }^{14}$ The high lateral portal is placed above the fat pad to optimize visualization for diagnostic arthroscopy and evaluation of the tibial insertion. A spinal needle is used to locate the appropriate position of the central and accessory medial portals to provide the proper trajectory and visualization.

\section{Intraoperative Evaluation/Decision Making}

The patient's native anatomy is preserved by careful dissection of the native tibial insertion. Both anteromedial (AM) and PL bundles are identified using a thermal device (Arthrocare, Austin, TX), and mea-
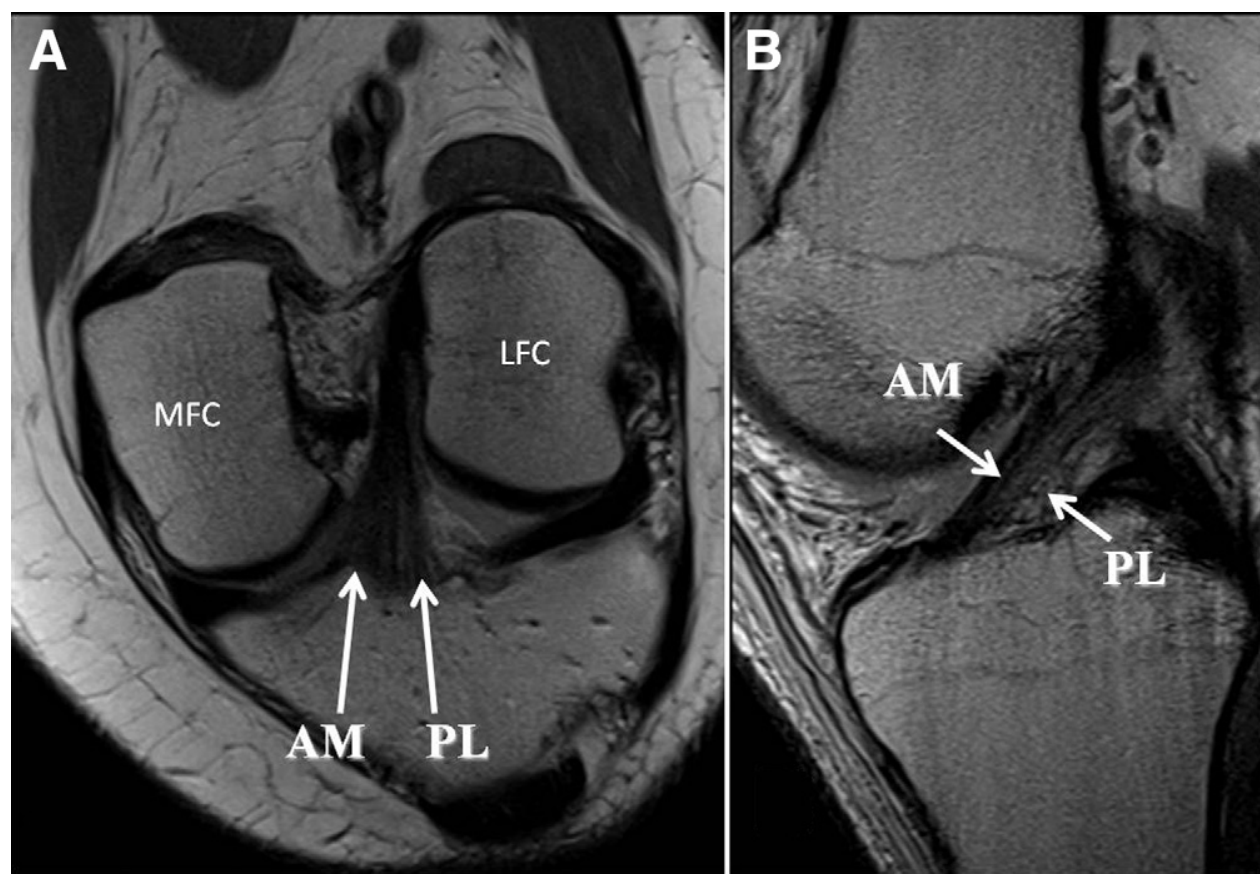

Figure 1. Oblique magnetic resonance imaging sequences oriented in same plane as ACL. (A) Coronal oblique image of ACL. (B) Sagittal oblique image of ACL. (AM, anteromedial bundle; LFC, lateral femoral condyle, where ACL inserts; MFC, medial femoral condyle; PL, posterolateral bundle.) 
Figure 2. Preoperative magnetic resonance imaging measurements. (A) Tibial insertion site length. (B) ACL inclination angle. (C) ACL length. (D) Quadriceps tendon thickness and patellar tendon thickness.

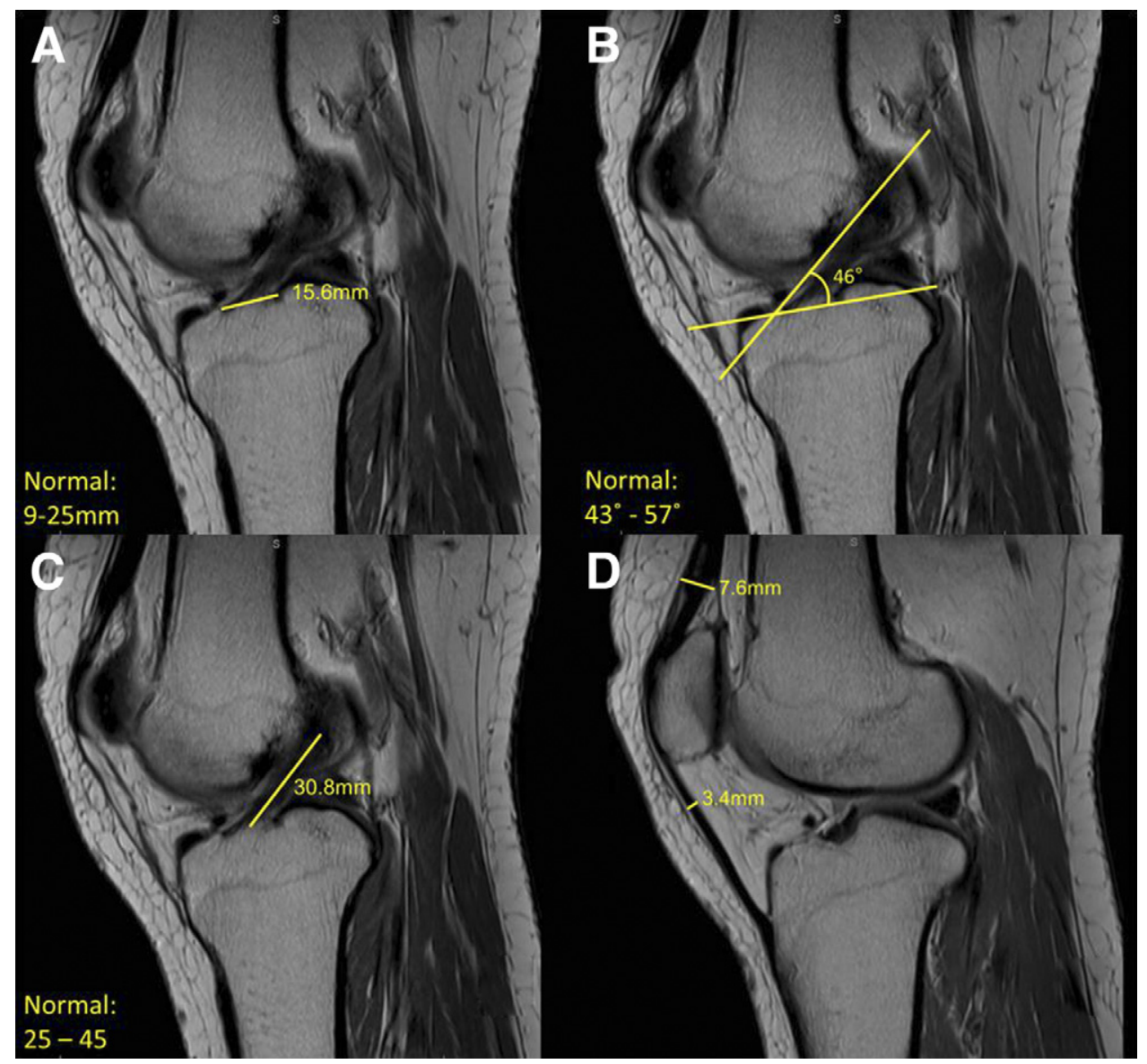

surements of tibial footprint length and width are obtained using a flexible, reusable, arthroscopic ruler (Smith \& Nephew, Andover, MA) (Fig 4). In patients with a tibial insertion site length of $14 \mathrm{~mm}$ or less, the native anatomy may be better reproduced with an SB

\section{Table 1. Key Points in Performing Anatomic} ACL Reconstruction

Patient positioning is critical to allow for knee hyperflexion during femoral tunnel preparation.

The 3-portal technique allows for thorough visualization of the native ACL anatomy.

Preparation of the femoral tunnel through the medial portal allows for accurate tunnel placement.

Notchplasty is not necessary for visualization and eliminates anatomic landmarks.

Preservation of native landmarks can be accomplished with careful debridement with a thermal device.

Careful measurement of notch and ACL insertion dimensions allows for individualized reconstruction.

Individualized surgery requires an appreciation of each patient's unique ACL structure and bony anatomy. reconstruction. Those with an insertion site length of 14 to $18 \mathrm{~mm}$ may undergo ACL-R by either an SB or DB technique. In patients with a tibial insertion length greater than $18 \mathrm{~mm}$, a DB ACL-R is recommended (Table 2). Similarly, the technique used may be dictated by dimensions of the femoral notch because a shallow or narrow notch may not accommodate a larger graft. Measurements of notch width and height are obtained using a flexible, reusable arthroscopic ruler (Smith \& Nephew). We recommend SB ACL-R in patients whose notch width is less than $12 \mathrm{~mm}$ (Table 2). By individualizing our technique, we may be able to more thoroughly reconstruct the ACL to accommodate the patient's individual needs.

Visualization of the femoral insertion is best achieved through the central and accessory medial portals (Table 1). The femoral insertion is obscured when one is viewing from the lateral portal. ${ }^{14}$ As such, a 3-portal technique negates the role of notchplasty to improve visualization. During identification and characterization of the femoral insertion, the knee is main- 


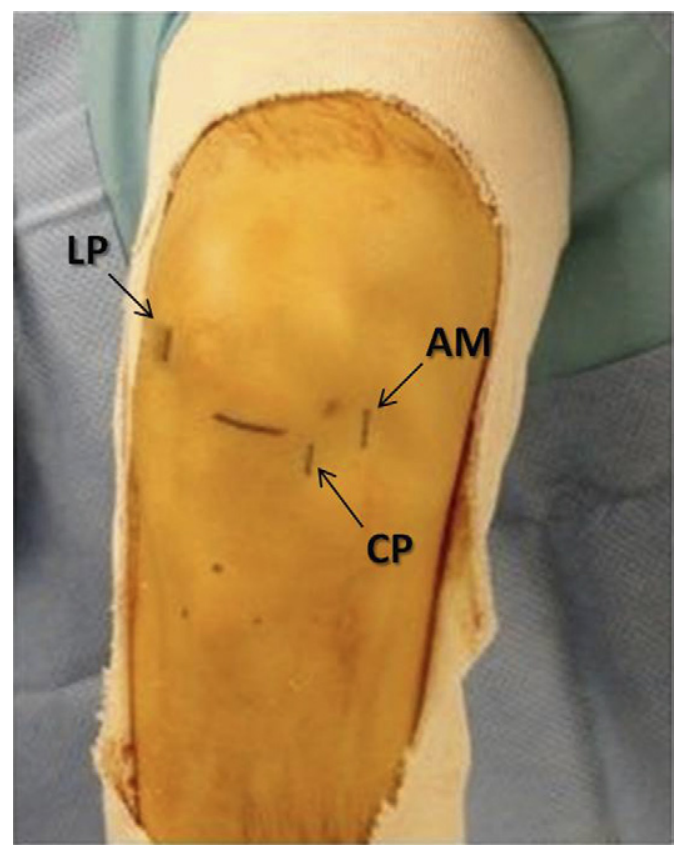

Figure 3. Three-portal arthroscopic technique. (CP, central portal; LP, high anterolateral portal; AM, accessory medial portal.)

tained in $90^{\circ}$ of flexion. Bony landmarks within the femoral notch help to identify the native ACL insertion. The lateral intercondylar ridge delineates the ACL insertion onto the femur, and the bifurcate ridge identifies the junction of the femoral AM and PL bundles. ${ }^{15}$

\section{Tunnel Preparation}

When one is using quadriceps tendon autograft, a single femoral tunnel is used at the center of the
TABLE 2. Algorithm for Determining Surgical Technique Based on Tibial Insertion Site Length and Notch Width

Recommended Repair Technique

\begin{tabular}{ll}
\hline $\begin{array}{l}\text { Tibial insertion length } \\
<14 \mathrm{~mm}\end{array}$ & SB reconstruction \\
$14-18 \mathrm{~mm}$ & SB or DB reconstruction \\
$>18 \mathrm{~mm}$ & DB reconstruction \\
Notch width & \\
$<12 \mathrm{~mm}$ & SB reconstruction \\
$>12 \mathrm{~mm}$ & SB or DB reconstruction \\
\hline
\end{tabular}

femoral ACL insertion. An awl is used to identify appropriate position between the AM and PL bundles (Fig 5). The knee is hyperflexed when the surgeon is using a straight guide pin and reamer to produce a tunnel of adequate length that does not violate the articular cartilage or place neurologic structures at risk. A preliminary tunnel is reamed to allow for assessment of the distance to the lateral cortex before enlarging the tunnel to the desired dimensions. In certain cases, flexible guide pins and reamers (Stryker, Kalamazoo, MI; Smith \& Nephew) can be beneficial because they produce a more spherical tunnel and do not require knee hyperflexion.

Next, tibial tunnel placement is addressed. A longitudinal incision is centered over the proximal aspect of the AM tibia. When a DB reconstruction is indicated, the tibial guide (Smith \& Nephew) is set at $45^{\circ}$ and placed in the center of the PL bundle for guide pin passage. The tibial guide is adjusted to $55^{\circ}$ and centered within the AM footprint for passage of the AM guide pin. Measurements are performed to assess for adequate pin spread (Fig 6). Graft size is taken into account when determining final tunnel dimensions. Tunnels are reamed

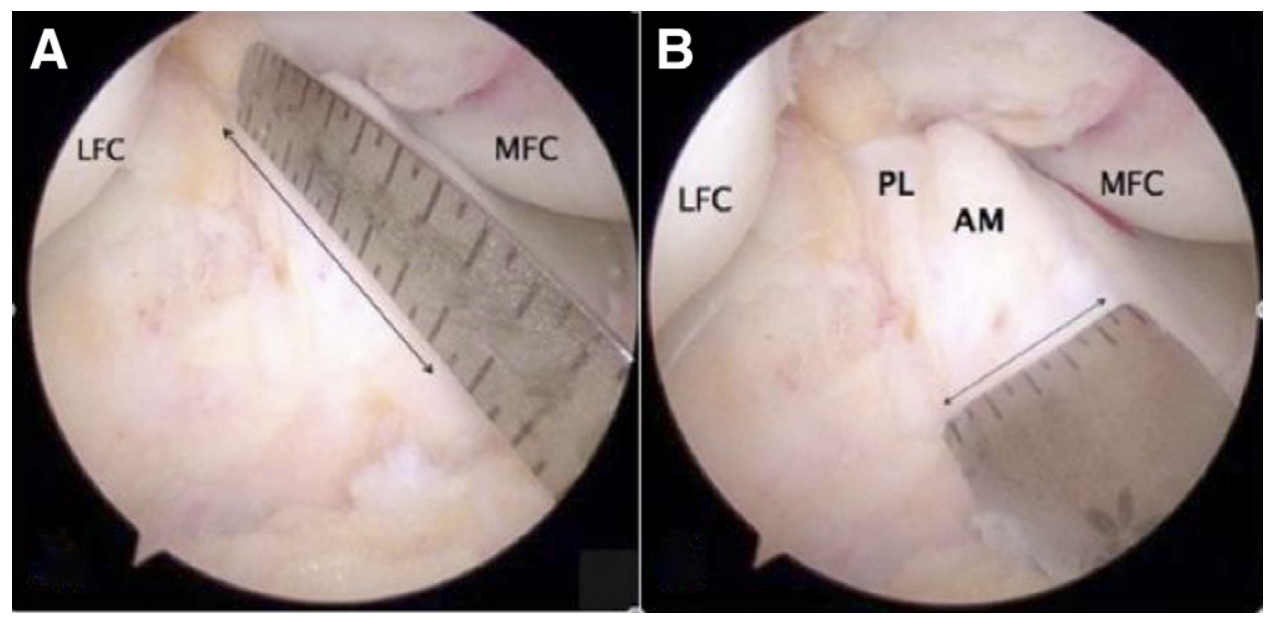

Figure 4. Tibial insertion site measurements by use of arthroscopic ruler. (A) Tibial insertion site length (anteroposterior). (B) Tibial insertion site width (mediolateral). (AM, anteromedial bundle; LFC, lateral femoral condyle; MFC, medial femoral condyle; PL, posterolateral bundle.) 
Figure 5. Single femoral tunnel placement. (A) Threedimensional computed tomography scan showing location of $\mathrm{AM}$ and PL bundles on medial wall of lateral femoral condyle (LFC). The red dot identifies the placement of the awl when one is performing an ACL-R using a single femoral tunnel. (B) Arthroscopic image of awl placement at desired femoral tunnel position. (AM, anteromedial bundle; PL, posterolateral bundle.)
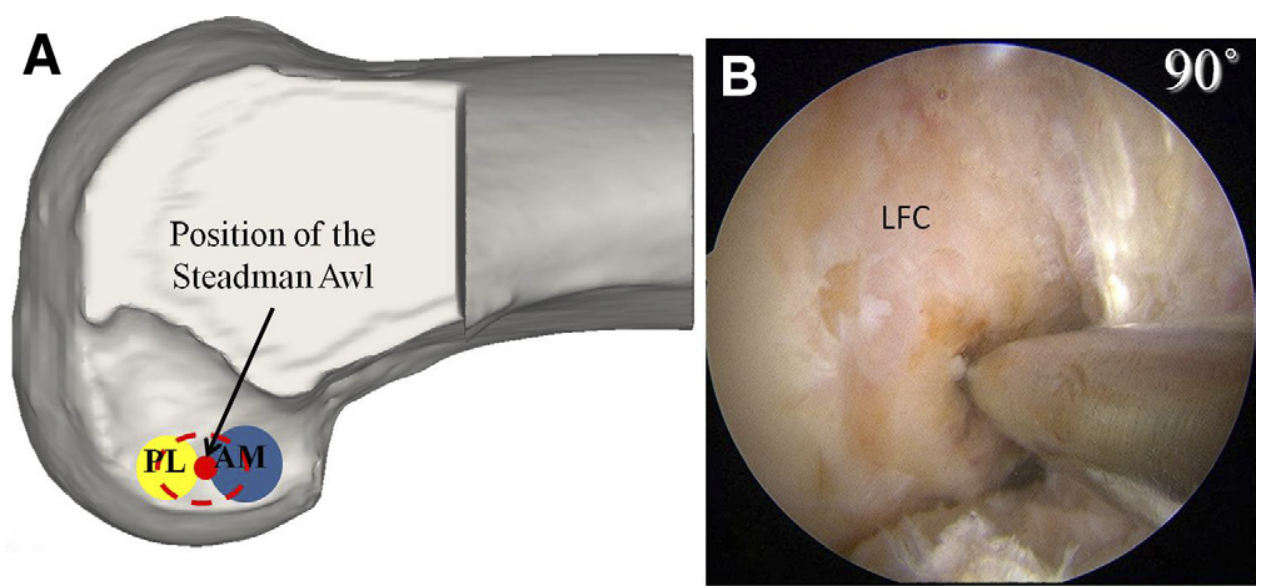

and dilated to the desired dimensions, and measurements of tunnel aperture are confirmed.

As discussed previously, in some cases the patient's anatomy is best reproduced with an SB reconstruction. Once the anatomic insertion of the AM and PL bundles is determined, a single guide pin is passed midway between the AM and PL tunnels. This tunnel is then reamed and dilated to the appropriate size. Measurements of tunnel aperture are confirmed.

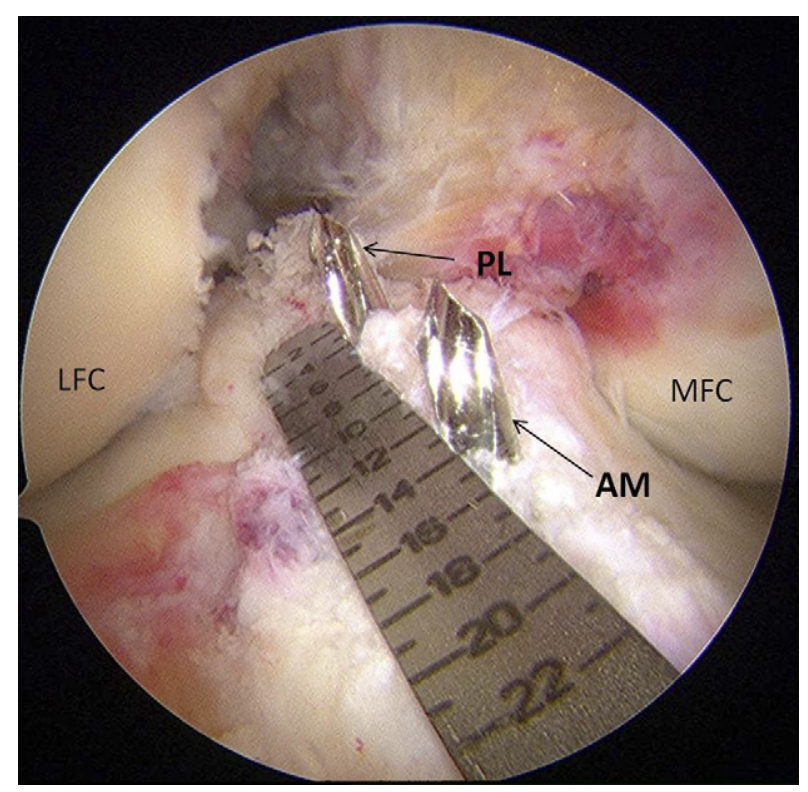

Figure 6. Pin spread measured with an arthroscopic ruler (Smith $\&$ Nephew). AM and PL guide pins are positioned in the center of the AM and PL tibial insertion sites. (AM, anteromedial guide pin; LFC, lateral femoral condyle; MFC, medial femoral condyle; PL, posterolateral guide pin.)

\section{Graft Passage and Fixation}

During graft passage for a proximal bone block, appropriate orientation is maintained through the accessory medial portal to prevent graft malposition within the femoral tunnel. Suspensory fixation (Endobutton, Smith $\&$ Nephew) is used on the femoral side, and position is confirmed with fluoroscopy. Flexible wires are passed retrograde through the appropriate tibial tunnels and retrieved through the central portal. The sutures for the PL tunnel are passed from the accessory medial tunnel through the notch and out the central portal. This suture is then passed through the loop of the flexible wire and out the PL tibial tunnel. Passage of the PL graft is monitored arthroscopically. The AM graft is passed in a similar fashion (Fig 7). The graft is tensioned and secured by interference screw (Smith \& Nephew) in full extension for the PL bundle and $45^{\circ}$ of flexion for the AM bundle. In cases where interference screw fixation may not be adequate, sutures may be tied over a post.

SB graft passage can be performed through the tibial tunnel in certain cases. Another option is to pass the SB graft in a similar fashion to a DB reconstruction. The bone block is passed through the accessory medial portal. Soft-tissue sutures are passed by flexible wires. The PL portion of an SB graft can be guided into its final position with a positioning suture placed within the graft. The PL position is manipulated and viewed intra-articularly before final fixation. The graft is then tensioned in $15^{\circ}$ to $20^{\circ}$ of flexion with interference screw fixation.

\section{Postoperative Rehabilitation}

Immediately after ACL-R, the patient is allowed to weight bear as tolerated with crutches and the brace locked in full extension. Home exercises are initiated 


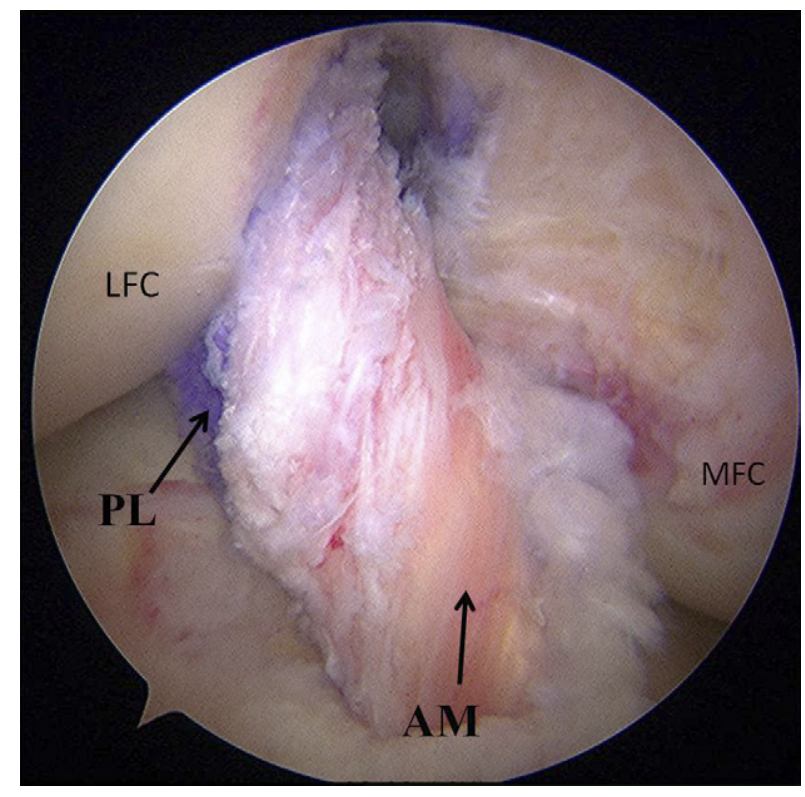

Figure 7. Arthroscopic view of anatomic ACL reconstruction. (AM, anteromedial bundle; LFC, lateral femoral condyle; MFC, medial femoral condyle; PL, posterolateral bundle.)

including quadriceps sets, straight-leg raises, calf pumps, and heel slides. Use of crutches is continued for the first month postoperatively. A systematic rehabilitation program is followed, and return to sport is expected between 9 and 12 months postoperatively.

\section{DISCUSSION}

There is increasing evidence indicating that the anatomic reconstruction of both bundles better restores normal knee kinematics, particularly internal and external rotation. ${ }^{16-21} \mathrm{~A}$ recent study by Hussein et al. ${ }^{18}$ showed that anatomic DB ACL-R better restored anteroposterior and rotational stability when compared with nonanatomic and anatomic SB ACL-R at 3- to 5-year follow-up. In addition, anatomic SB ACL-R showed improved stability when compared with nonanatomic SB ACL-R. Although this study shows improved stability with anatomic reconstruction, one limitation noted by the authors was that they did not account for individual variation in determining whether an SB or DB technique should be used. As previously mentioned, anatomic ACL-R is the recreation of the native ACL insertion site with respect to individual patient characteristics. In individuals with small insertion sites or narrow notches, a DB reconstruction may be technically compromising to surrounding knee structures. In such cases the DB concept can be achieved by an anatomic SB ACL-R.

It is also becoming clear that a "one-size-fits-all" approach does not adequately reproduce the native ACL. In fact, it appears that standard tunnels reproduce only a fraction of the native ACL. Kopf et al. ${ }^{22}$ recently described how tibial tunnel aperture varies with tunnel diameter and angle. This study showed that with standard drilling, only $57 \%$ of the native tibial insertion is reproduced. With respect to the femoral insertion, Hensler et al. ${ }^{23}$ showed that only $61 \%$ of the femoral insertion is reconstructed with standard tunnel preparation. By individualizing ACL-R, we may be able to reproduce more of the native anatomy and improve patient outcomes.

Only by gaining an appreciation of each patient's unique anatomy will it be possible to perform individualized, anatomic ACL-R. Our hope is that by closely examining the approach to anatomic ACL-R, we can improve the surgical technique and, most importantly, long-term patient outcomes. By individualizing ACL-R, we hope to eliminate nonanatomic ACL-R as a risk factor for osteoarthritis.

\section{REFERENCES}

1. Maletius W, Messner K. Eighteen- to twenty-four-year follow-up after complete rupture of the anterior cruciate ligament. Am J Sports Med 1999;27:711-717.

2. Kopf S, Forsythe B, Wong AK, et al. Nonanatomic tunnel position in traditional transtibial single-bundle anterior cruciate ligament reconstruction evaluated by three-dimensional computed tomography. J Bone Joint Surg Am 2010;92:14271431 .

3. Freedman KB, D'Amato MJ, Nedeff DD, Kaz A, Bach BR Jr. Arthroscopic anterior cruciate ligament reconstruction: A metaanalysis comparing patellar tendon and hamstring tendon autografts. Am J Sports Med 2003;31:2-11.

4. Biau DJ, Katsahian S, Nizard R. Hamstring tendon autograft better than bone-patellar tendon-bone autograft in ACL reconstruction-A cumulative meta-analysis and clinically relevant sensitivity analysis applied to a previously published analysis. Acta Orthop 2007;78:705-707; author reply 707-708.

5. Crawford C, Nyland J, Landes S, et al. Anatomic double bundle ACL reconstruction: A literature review. Knee Surg Sports Traumatol Arthrosc 2007;15:946-964; discussion 945.

6. Tashman S, Collon D, Anderson K, Kolowich P, Anderst W. Abnormal rotational knee motion during running after anterior cruciate ligament reconstruction. Am J Sports Med 2004;32: 975-983.

7. Ristanis S, Giakas G, Papageorgiou CD, Moraiti T, Stergiou $\mathrm{N}$, Georgoulis $\mathrm{AD}$. The effects of anterior cruciate ligament reconstruction on tibial rotation during pivoting after descending stairs. Knee Surg Sports Traumatol Arthrosc 2003;11:360365.

8. Lohmander LS, Ostenberg A, Englund M, Roos H. High prevalence of knee osteoarthritis, pain, and functional limita- 
tions in female soccer players twelve years after anterior cruciate ligament injury. Arthritis Rheum 2004;50:3145-3152.

9. Woo SL, Kanamori A, Zeminski J, Yagi M, Papageorgiou C, $\mathrm{Fu} \mathrm{FH}$. The effectiveness of reconstruction of the anterior cruciate ligament with hamstrings and patellar tendon. A cadaveric study comparing anterior tibial and rotational loads. J Bone Joint Surg Am 2002;84:907-914.

10. Yagi M, Wong EK, Kanamori A, Debski RE, Fu FH, Woo SL. Biomechanical analysis of an anatomic anterior cruciate ligament reconstruction. Am J Sports Med 2002;30:660-666.

11. van Eck CF, Lesniak BP, Schreiber VM, Fu FH. Anatomic single- and double-bundle anterior cruciate ligament reconstruction flowchart. Arthroscopy 2010;26:258-268.

12. Harris NL, Smith DA, Lamoreaux L, Purnell M. Central quadriceps tendon for anterior cruciate ligament reconstruction. Part I: Morphometric and biomechanical evaluation. Am J Sports Med 1997;25:23-28.

13. Stäubli HU, Schatzmann L, Brunner P, Rincón L, Nolte LP. Mechanical tensile properties of the quadriceps tendon and patellar ligament in young adults. Am J Sports Med 1999;27:27-34.

14. Cohen SB, Fu FH. Three-portal technique for anterior cruciate ligament reconstruction: Use of a central medial portal. Arthroscopy 2007;23:e321-e325.

15. Ferretti M, Ekdahl M, Shen W, Fu FH. Osseous landmarks of the femoral attachment of the anterior cruciate ligament: An anatomic study. Arthroscopy 2007;23:1218-1225.

16. Aglietti P, Giron F, Cuomo P, Losco M, Mondanelli N. Singleand double-incision double-bundle ACL reconstruction. Clin Orthop Relat Res 2007;454:108-113.
17. Järvelä T. Double-bundle versus single-bundle anterior cruciate ligament reconstruction: A prospective, randomize clinical study. Knee Surg Sports Traumatol Arthrosc 2007;15:500-507.

18. Hussein M, van Eck CF, Cretnik A, Dinevski D, Fu FH. Prospective randomized clinical evaluation of conventional single-bundle, anatomic single-bundle, and anatomic double-bundle anterior cruciate ligament reconstruction: 281 cases with 3- to 5-year follow-up. Am J Sports Med. 15 November, 2011. [Epub ahead of print.]

19. Muneta T, Koga H, Mochizuki T, et al. A prospective randomized study of 4-strand semitendinosus tendon anterior cruciate ligament reconstruction comparing single-bundle and double-bundle techniques. Arthroscopy 2007;23:618-628.

20. Yasuda K, Kondo E, Ichiyama H, Tanabe $\mathrm{Y}$, Tohyama $\mathrm{H}$. Clinical evaluation of anatomic double-bundle anterior cruciate ligament reconstruction procedure using hamstring tendon grafts: Comparisons among 3 different procedures. Arthroscopy 2006;22:240-251.

21. Siebold R, Dehler C, Ellert T. Prospective randomized comparison of double-bundle versus single-bundle anterior cruciate ligament reconstruction. Arthroscopy 2008;24:137145 .

22. Kopf S, Pombo MW, Szczodry M, Irrgang JJ, Fu FH. Size variability of the human anterior cruciate ligament insertion sites. Am J Sports Med 2011;39:108-113.

23. Hensler D, Working ZM, Illingworth KD, Thorhauer ED, Tashman S, Fu FH. Medial portal drilling: Effects on the femoral tunnel aperture morphology during anterior cruciate ligament reconstruction. J Bone Joint Surg Am 2011;93:2063-2071. 